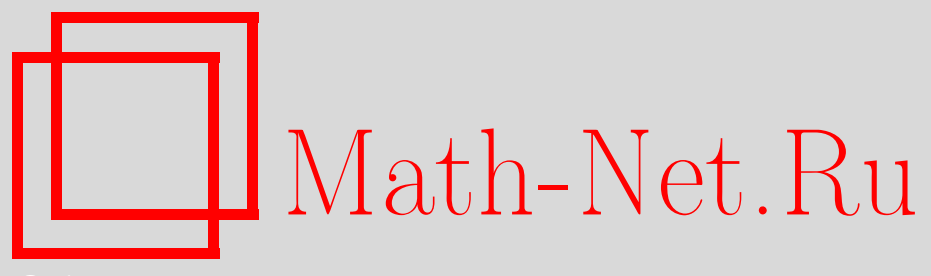

А. В. Комлов, С. П. Суетин, Формула Видома для старшего коэффициента полинома, ортонормированного относительно переменного веса, УМН, 2012, том 67, выпуск $1,183-184$

DOI: https://doi.org/10.4213/rm9462

Использование Общероссийского математического портала Math-Net.Ru подразумевает, что вы прочитали и согласны с пользовательским соглашением http://www . mathnet.ru/rus/agreement

Параметры загрузки:

IP: 3.89 .185 .249

26 апреля 2023 г., $17: 57: 47$

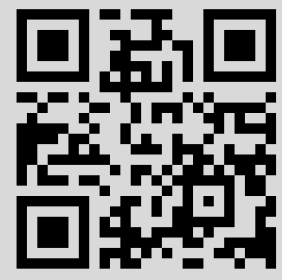




\title{
Формула Видома для старшего коэффициента полинома, ортонормированного относительно переменного веса
}

\author{
А. В. Комлов, С. П. Суетин
}

1. Пусть задан полином $Q(x)=x^{2 m}+\cdots$ степени $2 m$ с вещественными коэффициентами - потенциал внешнего поля в стандартной теоретико-потенциальной задаче (1) (см. [1]-[3]). Пусть $E=\bigsqcup_{j=1}^{p} E_{j}$, где $E_{j}=\left[e_{2 j-1}, e_{2 j}\right], j=1, \ldots, p,-$ система отрезков на вещественной прямой $\mathbb{R}$, удовлетворяющая следующему условию. В классе $M_{1}(E)$ единичных мер $\mu$ с носителями $\operatorname{supp} \mu$ на $E$ (единственное) решение $\lambda=\lambda_{Q} \in M_{1}(E)$ экстремальной задачи

$$
I_{Q}(\lambda)=\min _{\mu \in M_{1}(E)} I_{Q}(\mu)
$$

обладает следующими свойствами: $\operatorname{supp} \lambda=E$ (т. е. мера $\lambda$ заполняет весь компакт $E$ ) и $d \lambda(x)=h(x) / \sqrt{\left|H_{2 p}(x)\right|} d x, x \in E$, где функция $h$ голоморфна и отлична от нуля на $E, H_{2 p}(z)=\left(z-e_{1}\right) \cdots\left(z-e_{2 p}\right)$ (рассматривается арифметическое значение корня на $E)$. В (1) $I_{Q}$ - функционал энергии с полем $Q: I_{Q}(\mu)=\int\left(V^{\mu}(x)+2 Q(x)\right) d \mu(x)$, $V^{\mu}(x)=-\int \log |x-t| d \mu(t)$. Существование такой системы отрезков $E$ для заданного поля $Q$ вытекает из общих результатов работ [1], [4], [5]. Тем самым, здесь рассматривается случай так называемой "стенки" ("hard edge case"; см. [4], [6]). Экстремальная мера $\lambda$ является единственной равновесной мерой (в классе $M_{1}(E)$ ) во внешнем поле $Q: V^{\lambda}(x)+Q(x) \equiv w_{Q}, x \in E, w_{Q}-$ постоянная равновесия.

2. Пусть последовательность полиномов $P_{n}(x)=x^{n}+\cdots, n=1,2, \ldots$, определяется соотношениями ортогональности $\int_{E} P_{n}(x) x^{j} \varphi_{n}(x) d x=0, j=0,1, \ldots, n-1$, где $\varphi_{n}(x)$ - заданная последовательность весовых функций на $E$. Многочлен $P_{n}$ минимизирует норму $\int P_{n}^{2}(x) \varphi_{n}(x) d x$ в классе всех многочленов степени $n$ с единичным старшим коэффициентом; распределение нулей таких многочленов зависит от асимптотического поведения функций $\varphi_{n}$ при $n \rightarrow \infty$ (см. [1], [7]). В дальнейшем предполагается, что $\varphi_{n}(x)=e^{-2 n Q(x)} / \sqrt{\left|H_{2 p}(x)\right|} ;$ пусть $p_{n}(x, n)=\gamma_{n}(n) x^{n}+\cdots-$ соответствующая последовательность нормированных полиномов, $\gamma_{n}(n)>0$. Тогда [1]

$$
\left|p_{n}(z, n)\right|^{1 / n} \rightarrow e^{w_{Q}-V^{\lambda}(z)}, \quad n \rightarrow \infty,
$$

локально равномерно (т. е. на компактных подмножествах) в области $D=\overline{\mathbb{C}} \backslash E$. Соотношение (2) принято называть формулой слабой (или логарифмической) асимптотики для полиномов $p_{n}(\cdot, n)$; из (2) вытекает формула слабой асимптотики для старших коэффициентов: $\gamma_{n}(n)^{1 / n} \rightarrow e^{w_{Q}}, n \rightarrow \infty$. В теории случайных матриц (см. [6], [8]-[10]), естественным образом возникает задача о формулах сильной асимптотики для полиномов $p_{n}(z, n)$ и коэффициентов $\gamma_{n}(n)$. Для случая "soft edge case" такие формулы получены в работе [5]. В настоящей работе формула сильной асимптотики для $\gamma_{n}(n)$ получена для случая "стенки". Формула (3) аналогична классической формуле Видома $[11 ; \S 6$, теорема 6.2] для веса, не зависящего от $n$ (см. также [12]-[14]). Для одного отрезка случай "стенки" изучен в работе [15; теорема 2]; полученные в настоящей работе результаты обобщают результаты работы [15] на случай произвольного числа отрезков $p \geqslant 2$.

Работа выполнена при поддержке РФФИ (грант № 11-01-00330) и программы "Ведущие научные школы" (грант НШ-4664.2012.1). 
3. Пусть $g(z, \infty)=g_{D}(z, \infty)$ - функция Грина для области $D$ с особенностью в бесконечно удаленной точке $z=\infty, \omega_{k}(z)$ - гармоническая мера (в точке $z \in D$ ) отрезка $E_{k}$ относительно $\bigsqcup_{j \neq k} E_{j}, k=1, \ldots, p$.

Теорема 1. Пусть $E=\bigsqcup_{j=1}^{p}\left[e_{2 j-1}, e_{2 j}\right], \lambda \in M_{1}(E)$ - равновесная мера во внешнем поле $Q, w_{Q}$ - постоянная равновесия. Тогда для старшего коэффициента $\gamma_{n}(n)$ нормированного относительно веса $\varphi_{n}(x)=e^{-2 n Q(x)} / \sqrt{\left|H_{2 p}(x)\right|}, x \in E$, полинома справедлива следующая формула:

$$
\gamma_{n}(n)=e^{n w_{Q}} \exp \left\{-\frac{1}{2} \sum_{j=1}^{p-1} \varepsilon_{j} g\left(z_{j}(n), \infty\right)\right\}\left(1+O\left(\delta^{n}\right)\right), \quad \delta \in(0,1), \quad n \rightarrow \infty .
$$

Точки $z_{j}(n), z_{j}(n) \in\left[e_{2 j}, e_{2 j+1}\right]$ и параметры $\varepsilon_{j}= \pm 1, j=1, \ldots, p-1$, однозначно определяются из следующей системы сравнений ( $c_{k}$ - некоторые постоянные):

$$
\sum_{j=1}^{p-1} \varepsilon_{j} \omega_{k}\left(z_{j}\right)=-2 n \lambda\left(E_{k}\right)+c_{k} \quad(\bmod 2), \quad k=1, \ldots, p-1 .
$$

Из (3), (4) следует, что в типичном случае при $n \rightarrow \infty$ величина $2 \log \left(\gamma_{n}(n) e^{-n w_{Q}}\right)$ осииллирует около нуля и заполняет целый отрезок $[-A, A] ; A=\sum_{j=1}^{p-1} g\left(z_{j}^{*}, \infty\right), z_{j}^{*} \in$
$\left(e_{2 j}, e_{2 j+1}\right)$ - критические точки функции Грина.

Теорема 1 вытекает из формулы (5) сильной асимптотики для полиномов $p_{n}(x, n)$.

Теорема 2. Пусть $p_{n}(x, n)$ - полиномы, нормированные относительно веса $\varphi_{n}(x)=e^{-2 n Q(x)} / \sqrt{\left|H_{2 p}(x)\right|}, x \in E, a \Psi_{n}(z, n)$ - соответствующая нормированная пси-функиия Наттолла (см. [13], [14], [3]). Тогда справедлива следующал формула:

$$
p_{n}(z, n)=\Psi_{n}(z, n)\left(1+O\left(\delta^{n}\right)\right), \quad \delta \in(0,1), \quad z \in K, \quad n \rightarrow \infty,
$$

где $K$ - произвольный компакт в $\overline{\mathbb{C}}$, лежащий вне выпуклой оболочки компакта $E$.

Теорема 2 доказывается методом, основанным на сингулярном интегральном уравнении Наттолла (см. [16], [13], [15], [3]).

\section{Список литературы}

[1] А. А. Гончар, Е. А. Рахманов, Матем. сб., 125(167):1(9) (1984), 117-127. [2] H. N. Mhaskar, E. В. Saff, Trans. Amer. Math. Soc., 285:1 (1984), 203-234. [3] А. И. Аптекарев, В.И. Буслаев, А. Мартинес-Финкельштейн, С. П. Суетин, УМН, 66:6(402) (2011), 37-122. [4] В. С. Буяров, Е. А. Рахманов, Матем. сб., 190:6 (1999), 11-22. [5] P. Deift, T. Kriecherbauer, K. T.-R. McLaughlin, S. Venakides, X. Zhou, Comm. Pure Appl. Math., 52:11 (1999), 1335-1425. [6] L. Pastur, J. Approx. Theory, 139:1-2 (2006), 269-292. [7] A. А. Гончар, Е. А. Рахманов, Матем. сб., 134(176):3(11) (1987), 306-352. [8] А. И. Аптекарев, В. Г. Лысов, Д. Н. Туляков, Матем. сб., 202:2 (2011), 3-56. [9] А. И. Аптекарев, А.Э. Койэлаарс, УМH, 66:6(402) (2011), 123-190. [10] Л. Эрдёш, УМН, 66:3(399) (2011), 67-198. [11] H. Widom, Adv. Math., 3:2 (1969), 127-232. [12] А. И. Аптекарев, Матем. сб., 125(167):2(10) (1984), 231-258. [13] С. П. Суетин, Матем. сб., 191:9 (2000), 81-114. [14] С. П. Суетин, Совр. пробл. матем., 6, МИАН, М., 2006, 3-74. [15] A. I. Aptekarev, W. Van Assche, J. Approx. Theory, 129:2 (2004), 129-166. [16] J. Nuttall, Constr. Approx., 6:2 (1990), 157-166.

\section{А. В. Комлов (А. V. Komlov)}

Математический институт им. В. А. Стеклова РАН

E-mail: komlov@mi.ras.ru

\section{C. П. Суетин (S. P. Suetin)}

Математический институт им. В. А. Стеклова РАН

E-mail: suetin@mi.ras.ru
Представлено А. Г. Сергеевым Принято редколлегией 10.01.2012 